\title{
Premicellar aggregation of amphiphilic molecules: Aggregate lifetime and polydispersity
}

\author{
Radina Hadgiivanova and Haim Diamant* \\ School of Chemistry, Raymond 8 Beverly Sackler Faculty of Exact Sciences, Tel Aviv University, Tel Aviv 69978, Israel
}

(Dated: January 29, 2009)

\begin{abstract}
A recently introduced thermodynamic model of amphiphilic molecules in solution has yielded, under certain realistic conditions, a significant presence of metastable aggregates well below the critical micelle concentration - a phenomenon that has been reported also experimentally. The theory is extended in two directions pertaining to the experimental and technological relevance of such premicellar aggregates. (a) Combining the thermodynamic model with reaction rate theory, we calculate the lifetime of the metastable aggregates. (b) Aggregation number fluctuations are examined. We demonstrate that, over most of the metastable concentration range, the premicellar aggregates should have macroscopic lifetimes and small polydispersity.
\end{abstract}

PACS numbers: 82.70.Uv,64.60.My,64.60.an,64.75.Yz

\section{INTRODUCTION}

The natural and technological applications of self-assembled amphiphilic structures (micelles) in aqueous solution are vast [1, 2]. According to the common view, supported by numerous macroscopic experiments (e.g., conductivity and surface-tension measurements) and widely accepted theories [2, 3], amphiphilic molecules form aggregates above a well defined critical micelle concentration ( $\mathrm{cmc}$ ). During the years, however, there have been several experimental indications [4, 5, 6, 7, 8], as well as theoretical ones [9], for the appearance of aggregates at concentrations well below the $\mathrm{cmc}$ - a phenomenon referred to as premicellar aggregation. In particular, a fluorescence correlation spectroscopy experiment [6] seems to have provided direct observation of premicellar aggregates at concentrations four times lower than the macroscopically determined cmc.

Recently we have presented a two-state (monomer-aggregate) thermodynamic model for amphiphilic aggregation which, alongside its simplicity, allows the study of metastable micelles of variable size [10]. The analysis has yielded a sequence of three well separated concentrations: $c_{1}$, where a metastable aggregated state appears but is not significantly occupied; $c_{2}$, above which an appreciable amount of metastable aggregates forms; and $c_{3}$, where the aggregated state becomes stable. The cmc as commonly measured in macroscopic experiments has been shown to correspond to $c_{3}$. Thus, appreciable premicellar aggregation may occur in the concentration range between $c_{2}$ and $c_{3}$. We have shown that, so long as the micelles are not too large, the extent of premicellar aggregation is much larger than what would be expected from mere finite-size effects. This somewhat surprising effect stems from the variability of the "excited state", i.e., the freedom of the micelles to select their sizes, and the small free-energy difference between the pure monomeric state and the metastable one, which contains mostly monomers and a low concentration of aggregates. In addition, we have found that the premicellar regime is characterized by a weak concentration dependence of micelle size. Thus, the premicellar aggregates have roughly the same size as those observed above the $\mathrm{cmc}$, in agreement with the experiment of Ref. 6].

The analysis in Ref. [10], which is purely thermodynamic, does not fully account for possible kinetic barriers for premicellar aggregation. It has been assumed, and will be assumed below, that the solution has fully equilibrated. (In cases where high nucleation barriers exist, they might be overcome in practice, e.g., through heterogeneous nucleation.) Two key issues remain open, however. First, while the metastable premicellar state may be appreciably occupied at equilibrium, the aggregates might be short-lived. Second, although the mean size of the premicellar aggregates is similar to that of the micelles above the cmc, the size distribution in the former case might be much broader. Evidently, these issues of lifetime and polydispersity could jeopardize the experimental and technological relevance of premicellar aggregation.

Micellization dynamics (above the cmc) were thoroughly studied in previous works, both experimentally (see Ref. 11] and references therein) and theoretically (see, e.g., Refs. 12, 13, 14, 15, 16, 17, 18, 19, 20]). Two disparate time scales are involved in the dynamics, corresponding to the exchange of individual monomers between the micelle and the solution and the much slower process of micelle formation and breakup. Being interested in aggregate stability, we

*Electronic address: hdiamant@tau.ac.il 
focus here on the latter. We use the free energy landscape, as obtained from the thermodynamic model [10], within Kramers' rate theory [21, 22] to study the lifetime of premicellar aggregates. The second extension of the theory is an examination of aggregate size fluctuations in the premicellar regime.

In Sec. [I] we briefly review the thermodynamic model of Ref. [10] and then extend it to study lifetime and polydispersity. Representative numerical results are presented in Sec. III. In Sec. IV] we discuss the results and their implications for the observation of premicellar aggregates.

\section{MODEL}

\section{A. Free energy}

Our starting point is the two-state thermodynamic model of micellization presented in detail in Ref. [10]. The solution, containing a volume fraction $\phi$ of amphiphiles, is assumed to consist of two species: monomers with volume fraction $\phi_{1}$, and aggregates of $m$ molecules with volume fraction $\left(\phi-\phi_{1}\right)$. The volume fraction of water is $(1-\phi)$. Both $\phi_{1}$ and $m$ are treated as degrees of freedom, i.e., the system can select the number of aggregates as well as their size, while the total volume fraction $\phi$ is the control parameter. The two-state approximation restricts the validity of the entire approach to compact (spherical) micelles, whose size distribution is relatively narrow [2]. The free energy of the solution contains a mixing-entropy contribution and an interaction term. The former is calculated using a coarse-grained (Flory-Huggins) lattice scheme, where a water molecule occupies a single lattice site (of volume $a^{3}$ ), and each amphiphile occupies $n$ sites. The latter term, containing all other contributions to the free energy of transfer of a monomer from the solution to an aggregate of size $m$, is represented by a single phenomenological function, $u(m)$. The resulting free-energy density (per lattice site) is

$$
F_{\mathrm{S}}\left(\phi_{1}, m, \phi\right)=\frac{\phi_{1}}{n} \ln \phi_{1}+\frac{\phi-\phi_{1}}{n m}\left[\ln \left(\phi-\phi_{1}\right)-m u(m)\right]+(1-\phi) \ln (1-\phi) .
$$

(All energies in this paper are expressed in units of the thermal energy $k_{\mathrm{B}} T$.) The specific form of $u(m)$ is not crucial for the analysis; it should merely have a maximum at a finite value of $m$ to ensure the formation of finite aggregates (rather than a macroscopic phase) upon increasing $\phi$. For the sake of numerical examples we shall use the following function [10, 23]:

$$
u(m)=u_{0}-\sigma m^{-1 / 3}-\kappa m^{2 / 3} .
$$

(The physical origins of the terms appearing in Eq. (2), as well as the rather limited range of relevant values for the parameters $u_{0}, \sigma$, and $\kappa$, are discussed in Ref. [10].)

Equation (10) defines a free-energy landscape over a two-dimensional space of macrostates $\left(\phi_{1}, m\right)$. Along the $\phi_{1}$ axis $F_{\mathrm{S}}$ is always convex, i.e., it has a single minimum at $\phi_{1}^{\min }(m, \phi)$ for all values of $m$ and for any $\phi$ [10]. Along the $m$ axis the free energy becomes nonconvex above a certain volume fraction, $\phi>c_{1}$, with two minima at $m=1$ and $m^{\min }$, and a maximum in-between, at $m=m^{\max }$. In the premicellar regime of interest, $c_{1}<c_{2}<\phi<c_{3}$, the free energy has a global minimum still at the pure monomeric state, $\left[\phi_{1}^{\min }(m=1), m=1\right]$, as well as a local minimum at the metastable aggregated state, $\left[\phi_{1}^{\min }\left(m^{\mathrm{min}}\right), m^{\mathrm{min}}\right]$ (containing mostly monomers and a a low concentration of aggregates). The two minima are separated by the saddle point $\left[\phi_{1}^{\min }\left(m^{\max }\right), m^{\max }\right]$, which poses a kinetic barrier for the disintegration of the metastable aggregated state into the stable monomeric one.

The following analysis relies on two basic assumptions. First, we assume that overcoming the barrier at the saddle point $\left[\phi_{1}^{\min }\left(m^{\max }\right), m^{\max }\right]$ is the rate-limiting process in aggregate dissociation, whereas diffusion is much faster. Hence, the dynamics depend on $m$ alone, advancing at all times $t$ along the path $\left[\phi_{1}^{\min }(m(t)), m(t)\right]$. The second assumption arises from the necessity to relate our coarse-grained model with single-aggregate properties. Since the model [e.g., Eq. (1)] does not explicitly consider single aggregates but rather macrostates containing both monomers and aggregates, we shall consider, instead, a fictitious subsystem, of volume $V_{1}$, which on average contains a single aggregate of size $m^{\mathrm{min}}$. The volume of the aggregate itself is $n a^{3} \mathrm{~m}^{\mathrm{min}}$, and the volume fraction of aggregates is $\phi-\phi_{1}$. Hence, the subsystem volume is

$$
V_{1}(\phi)=\frac{n a^{3} m^{\min }(\phi)}{\phi-\phi_{1}^{\min }\left(m^{\min }(\phi)\right)} .
$$

Since $\phi-\phi_{1}$ is very small, $V_{1}$ is far from being microscopic, and we may apply our coarse-grained description to the subsystem, writing its free energy as

$$
F\left(\phi_{1}, m, \phi\right)=\frac{V_{1}(\phi)}{a^{3}} F_{\mathrm{s}},
$$


where $F_{\mathrm{s}}$ is given by Eq. (1). Thus, the dissociation of a single premicellar aggregate is treated as the transition of a mesoscopic subsystem from a metastable state, containing monomers and (on average) one aggregate, to the stable, purely monomeric state. For brevity the free energy of the subsystem along the dissociation path $\left[\phi_{1}^{\min }(m(t)), m(t)\right]$ is hereafter referred to as $F(m)$.

\section{B. Aggregate lifetime}

We follow the lines of Kramers' theory [21, 22] while adapting it to the case of premicellar aggregates. The main assumptions of this approach are as follows. (i) The energy barrier between the two states is sufficiently high, leading to separation of time scales between the fast monomer exchange process and the much slower aggregate dissociation. (ii) The free energy of the final (monomeric) state is much lower than that of the initial (aggregated) one, ensuring a practically unidirectional probability current from the aggregated to the monomeric state. The first assumption breaks down when $\phi$ is too small, i.e., as it gets too close to $c_{1}$; in the examples of Sec. III it becomes invalid already for $\phi \simeq c_{2}$. The second assumption fails when $\phi$ gets close to $c_{3}$. Thus, the following calculation is strictly valid only for $c_{2} \ll \phi \ll c_{3}$. (The behavior outside this domain of validity will be commented on separately in Sec. IV]) In addition, we assume that the aggregation number is large, $m \gg 1$, so that the discrete changes in $m$ can be replaced to a good approximation by continuous, infinitesimal ones.

We begin with the master equation for the probability density function, $f(m, t)$, of finding the subsystem around the state $\left[\phi_{1}^{\min }(m), m\right]$ at time $t$,

$$
\frac{\partial f(m, t)}{\partial t}=\int d k W(m-k, k) f(m-k, t)-\int d k W(m, k) f(m, t) d k,
$$

where $W(m, k)$ is the transition probability per unit time for the aggregation number to change from $m$ to $m+k$. Assuming that large jumps in aggregation number are improbable, we expand the first integral in Eq. (5) to second order in small $k$ and get the Fokker-Planck equation,

$$
\frac{\partial f}{\partial t}=-\frac{\partial j}{\partial m}, \quad j(m, t)=A(m) f(m, t)-\frac{\partial}{\partial m}[D(m) f(m, t)] .
$$

The first term in the probability current density $j$ describes a drift along the aggregation-number axis, with velocity $A(m)=\int d k k W(m, k)$. The second term represents diffusion along that axis, with a diffusion coefficient

$$
D(m)=\frac{1}{2} \int d k k^{2} W(m, k) .
$$

Demanding that $f$ reduce at equilibrium (i.e., when $j=0$ ) to the Boltzmann distribution, $f_{\mathrm{eq}}(m) \sim e^{-F(m)}$, one gets from Eq. (6) a generalized Einstein relation between $A$ and $D$,

$$
A(m)=-D(m) F^{\prime}(m)+D^{\prime}(m)
$$

where a prime denotes a derivative with respect to $m$. Substituting this relation back in Eq. (6), we rewrite the probability current density as

$$
j=-D(m) e^{-F(m)}\left[f(m, t) e^{F(m)}\right]^{\prime} .
$$

Thanks to the assumed high free-energy barrier, and the resulting separation of time scales, steady state can be assumed practically throughout the entire dissociation process. Thus, $\partial f / \partial t=\partial j / \partial m=0$, i.e., $j=j_{\text {ss }}$ independent of $m$. Equation (9) can then be integrated over $m$,

$$
j_{\mathrm{ss}} \int_{1}^{m^{\mathrm{min}}} d m \frac{e^{F}}{D}=-\left.f e^{F}\right|_{1} ^{m^{\mathrm{min}}} .
$$

The second assumption, of a large free-energy difference between the two states, implies that the right-hand side (rhs) of Eq. (10) is dominated by its value at $m^{\min }$. In addition, we assume that the subsystem is still mostly in the aggregated state near $m^{\min }$, at quasi-equilibrium, and, hence, $f(m, t) \sim e^{-F(m)}$. Expanding about $m^{\min }$ we obtain for the normalized probability density,

$$
f(m) \simeq\left[F^{\prime \prime}\left(m^{\mathrm{min}}\right) /(2 \pi)\right]^{1 / 2} e^{-\frac{1}{2} F^{\prime \prime}\left(m^{\mathrm{min}}\right)\left(m-m^{\mathrm{min}}\right)^{2}} .
$$


The rhs of Eq. (10) is given, therefore, by $-\left[F^{\prime \prime}\left(m^{\min }\right) /(2 \pi)\right]^{1 / 2} e^{F\left(m^{\mathrm{min}}\right)}$.

Treating the left-hand side (lhs) of Eq. (10) requires an estimate for the aggregation-number diffusion coefficient, $D(m)$. We use the definition of this coefficient, Eq. (7), together with Langer's formula for the transition probability [24],

$$
W(m, k) \sim \tau_{0}^{-1} e^{-k^{2} /(2 \Delta)} e^{-\frac{1}{2}[F(m+k)-F(m)]},
$$

where $\tau_{0}$ is a molecular time scale, and $\Delta$ is used to suppress large jumps in the aggregation number. Assuming that jumps much larger than unity are improbable, we set $\Delta=1$. We then expand $F(m+k)-F(m)$ in Eq. (12) to second order in $k$, normalize the transition probability, and substitute it in Eq. (7) to obtain

$$
D(m)=\frac{1}{2 \tau_{0}} \frac{4+F^{\prime 2}+2 F^{\prime \prime}}{\left(2+F^{\prime \prime}\right)^{2}} .
$$

Analysis of Eqs. (11)-(4) and (13) shows that for realistic aggregation numbers, $m \gg 1$, one has $|F| \gg|\ln D|$. Hence, the integral on the lhs of Eq. (10) is dominated by a small region around the maximum of $F$. We expand $F(m)$ about $m^{\max }$, integrate, and get for the lhs of Eq. (10), $j_{\mathrm{ss}}\left[2 \pi /\left|F^{\prime \prime}\left(m^{\max }\right)\right|\right]^{1 / 2} e^{F\left(m^{\max }\right) / D\left(m^{\max }\right)}$.

Substituting all these results in Eq. (10), we finally obtain for the micelle lifetime [25],

$$
\tau_{\mathrm{m}}=\left|j_{\mathrm{ss}}\right|^{-1}=\frac{4 \pi \tau_{0}}{\left(1+F^{\prime \prime}\left(m^{\max }\right) / 2\right)\left|F^{\prime \prime}\left(m^{\min }\right) F^{\prime \prime}\left(m^{\max }\right)\right|^{1 / 2}} e^{F_{\mathrm{b}}},
$$

where $F_{\mathrm{b}}=F\left(m^{\max }\right)-F\left(m^{\min }\right)$ is the height of the free-energy barrier between the aggregated and monomeric states. Equation (14), combined with Eqs. (1)-(4), yields the aggregate lifetime in the metastable, premicellar regime.

\section{Polydispersity}

For a given amphiphile volume fraction in the premicellar regime, $c_{2}<\phi<c_{3}$, the aggregation number of the metastable aggregates, $m^{\min }(\phi)$, is given by the local minimum of $F_{\mathrm{s}}$ of Eq. (11) [10]. To examine the polydispersity of the aggregates we should calculate the fluctuations of $m$ around $m^{\mathrm{min}}$ for a single aggregate. As explained in Sec. II A, within our coarse-grained framework we calculate, instead, the fluctuations of $m$ in a subsystem of volume $V_{1}$. The distribution of $m$ in that subsystem is given, for $m$ close to $m^{\mathrm{min}}$, by Eq. (11). Thus, we readily get for the mean-square size fluctuation,

$$
\left\langle\delta m^{2}\right\rangle=1 / F^{\prime \prime}\left(m^{\min }\right) .
$$

The relative width of the size distribution,

$$
w=\frac{\left\langle\delta m^{2}\right\rangle^{1 / 2}}{\langle m\rangle}=\frac{1}{m^{\min }\left[F^{\prime \prime}\left(m^{\min }\right)\right]^{1 / 2}},
$$

provides a convenient measure of the polydispersity.

\section{RESULTS}

We now demonstrate the results of the model in two numerical examples, representing two amphiphiles of differing hydrophobicity. The parameters of the amphiphiles are given in Table I amphiphile B being the more hydrophobic of the two. We use here the same two examples whose equilibrium properties have been thoroughly analyzed in Ref. [10]. Table \lists for these examples the volume-fraction bounds of the premicellar regime, $c_{2}$ and $c_{3}$, along with the aggregation numbers at these points, as obtained from the equilibrium theory.

In Table II we give the values of the free-energy barrier for aggregate dissociation at the lower and upper bounds of the premicellar regime, as calculated from Eqs. (1)-(4) using the parameters of Table I. At $\phi=c_{2}$ the barrier is negligible, of order $k_{\mathrm{B}} T$, yet, as $\phi$ increases through the premicellar regime, it becomes much larger than $k_{\mathrm{B}} T$. The resulting lifetimes, as calculated from Eq. (14), are given in Table II As an estimate for the molecular time scale we have used for both amphiphiles $\tau_{0}=10 \mathrm{~ns}$. (This is the diffusion time of a molecule, having a diffusion coefficient of $10^{-6} \mathrm{~cm}^{2} / \mathrm{s}$, along a distance of $1 \mathrm{~nm}$.) Corresponding to the increase in the free-energy barrier, the aggregate lifetime increases from milliseconds at the lower bound of the premicellar region to practically indefinite time. As 
TABLE I: Parameters and equilibrium properties of exemplary amphiphiles. $n$ - number of groups in hydrocarbon tail; $u_{0}$, $\sigma, \kappa$ - parameters of $u(m)$, the free energy of transfer in units of $k_{\mathrm{B}} T$ [Eq. (2)]. $c_{2}, c_{3}$ — volume-fraction bounds of the premicellar regime; $m^{\min }\left(c_{2}\right), m^{\min }\left(c_{3}\right)$ - aggregation numbers at these boundaries [10].

\begin{tabular}{ccccccccc}
\hline \hline amphiphile & $n$ & $u_{0}$ & $\sigma$ & $\kappa$ & $c_{2}$ & $c_{3}$ & $m^{\text {min }}\left(c_{2}\right)$ & $m^{\text {min }}\left(c_{3}\right)$ \\
\hline A & 13 & 10 & 11 & 0.08 & $8.0 \times 10^{-4}$ & $2.2 \times 10^{-3}$ & 53 \\
B & 20 & 14 & 14 & 0.05 & $1.6 \times 10^{-5}$ & $6.7 \times 10^{-5}$ & 118 & 128 \\
\hline \hline
\end{tabular}

TABLE II: Properties of premicellar aggregates. $F_{\mathrm{b}}$ - free-energy barrier for dissociation; $\tau_{\mathrm{m}}$ - aggregate lifetime; $w-$ relative width of size distribution; $c_{2}, c_{3}$ - lower and upper bounds of the premicellar regime. A value of $\tau_{0}=10 \mathrm{~ns}$ has been used for the molecular time scale.

\begin{tabular}{cccccc}
\hline \hline amphiphile & $F_{\mathrm{b}}\left(c_{2}\right)$ & $F_{\mathrm{b}}\left(c_{3}\right)$ & $\tau_{\mathrm{m}}\left(c_{2}\right)$ & $\tau_{\mathrm{m}}\left(c_{3}\right)$ & $w\left(c_{2}\right)$ \\
& $k_{\mathrm{B}} T$ & $k_{\mathrm{B}} T$ & $\mathrm{~s}$ & $\mathrm{~s}$ & $w\left(c_{3}\right)$ \\
\hline $\mathrm{A}$ & 1.3 & 30.4 & $2.0 \times 10^{-4}$ & $1.3 \times 10^{9}$ & 0.18 \\
$\mathrm{~B}$ & 0.5 & 112.5 & $4.3 \times 10^{-2}$ & $1.8 \times 10^{43}$ & 0.11 \\
\hline \hline
\end{tabular}

already noted in Sec. IIB our lifetime analysis is strictly valid only for $c_{2} \ll \phi \ll c_{3}$, and, hence, these values should be regarded merely as rough estimates.

The premicellar aggregate lifetime for amphiphile $\mathrm{A}$, scaled by the molecular time $\tau_{0}$, is depicted as a function of amphiphile volume fraction in Fig. 1. The roughly exponential increase of lifetime with concentration stems from the exponential dependence of $\tau_{\mathrm{m}}$ on the barrier height [Eq. (14)], which is the main source of concentration dependence. Two additional contributions to the dependence of $\tau_{\mathrm{m}}$ on $\phi$ are included in the prefactor of Eq. (14). The first, $\left(1+F^{\prime \prime}\left(m^{\max }\right) / 2\right)^{-1}$, comes from the aggregation-number diffusion coefficient, $D(m)$. This factor is practically concentration-independent, since in our examples the curvature of the saddle point is small, $\left|F^{\prime \prime}\left(m^{\max }\right)\right|<0.1$, and thus $D\left(m^{\max }\right) \simeq\left(2 \tau_{0}\right)^{-1}=$ const. The second pre-exponential factor in Eq. (14), $\left|F^{\prime \prime}\left(m^{\min }\right) F^{\prime \prime}\left(m^{\max }\right)\right|^{-1 / 2}$, depends on concentration primarily through $\left|F^{\prime \prime}\left(m^{\max }\right)\right|$, which is an increasing function of $\phi$. This factor causes the slightly weaker increase of lifetime with $\phi$ at small concentration (Fig. 1).

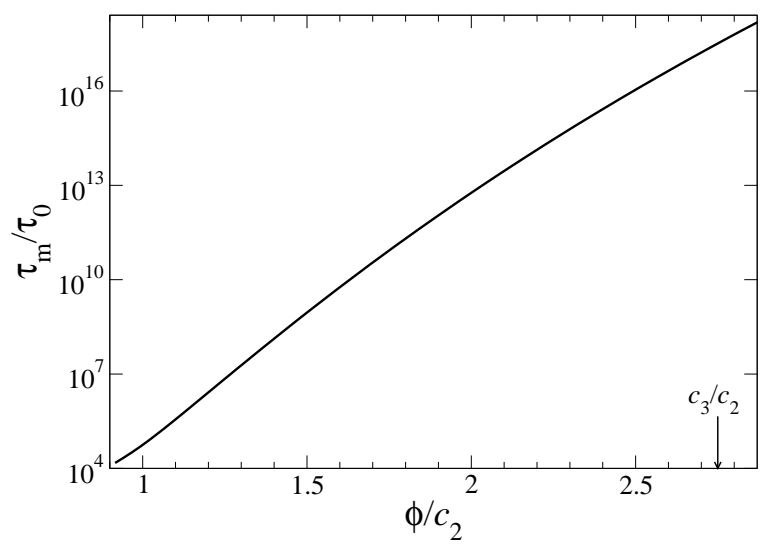

FIG. 1: Lifetime of premicellar aggregates of amphiphile A as a function of amphiphile volume fraction. The lifetime is scaled by the molecular time scale $\tau_{0}$ (of typical order of $10 \mathrm{~ns}$ ), and the volume fraction by $c_{2}$, the onset of premicellar aggregation. The volume fraction corresponding to the $\mathrm{cmc}\left(c_{3}\right)$ is indicated by an arrow. Parameters of amphiphile A are given in Table $\square$

In Fig. 1 we see that in the case of amphiphile A, assuming $\tau_{0} \sim 10 \mathrm{~ns}$, the aggregate lifetime reaches the order of 1 $\mathrm{s}$ for $\phi \simeq 1.4 c_{2}$, whereas the $\mathrm{cmc}$ is at $c_{3} \simeq 2.75 c_{2}$. Thus, the premicellar aggregates remain stable for a macroscopic time over a significant part of the premicellar regime. In the case of amphiphile B we find $\tau_{\mathrm{m}} \sim 1 \mathrm{~s}$ for $\phi \simeq 1.05 c_{2}$ while $c_{3} \simeq 4.2 c_{2}$, i.e., the aggregates are kinetically stable over a much larger portion (practically all) of the premicellar concentration range, as expected for a more hydrophobic amphiphile.

Figure 2 shows the mean-square fluctuation of the aggregation number for amphiphile A as a function of volume fraction. The corresponding relative width of the aggregate size distribution is presented in the inset. The polydispersity weakly decreases with concentration, i.e., the premicellar aggregates are nearly as monodisperse as the micelles 
above the cmc. In Table W we see that the same conclusions hold for amphiphile B. The small polydispersity (around $10 \%$ ), as well as the slightly increased value for the less hydrophobic amphiphile (A), are in agreement with the well known trends for spherical micelles above the $\mathrm{cmc}$, as established experimentally [11] and theoretically [2].

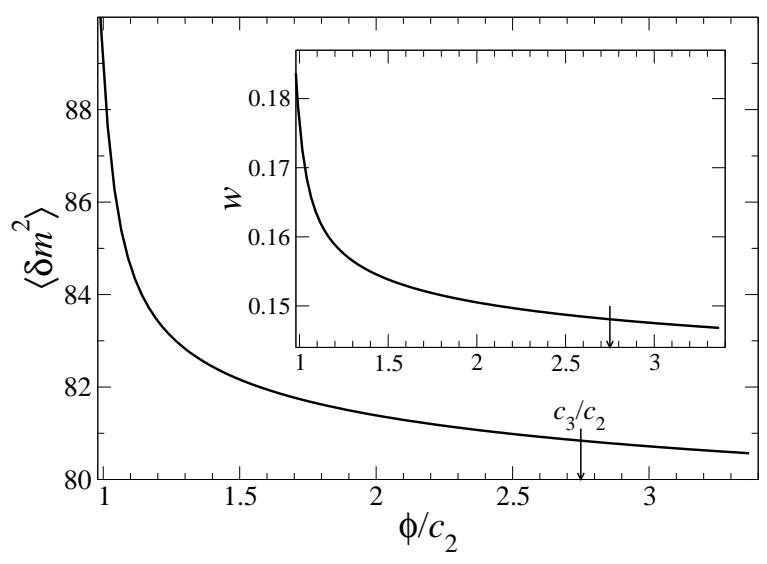

FIG. 2: Mean-square fluctuation of aggregation number for amphiphile A as a function of amphiphile volume fraction. The volume fraction is scaled by $c_{2}$, the onset of premicellar aggregation. The cmc $\left(c_{3}\right)$ is indicated by an arrow. The inset shows the relative width of the aggregate size distribution, $w=\left\langle\delta m^{2}\right\rangle^{1 / 2} /\langle m\rangle$. Parameters of amphiphile A are given in Table $\llbracket$

\section{DISCUSSION}

It has been shown in Sec. III that considerations of aggregate lifetime can reduce the concentration range in which premicellar aggregates may be experimentally observable and technologically relevant, compared to the range determined from equilibrium considerations alone [10]. In other words, the apparent concentration, above which an appreciable amount of metastable micelles appears, may be higher than $c_{2}$. We have demonstrated, nonetheless, that kinetic stability (i.e., macroscopic lifetime) still exists in most of the premicellar region. The more hydrophobic the surfactant, the wider the range of stability is. These conclusions are in line with results presented in Ref. [20]. Although that study does not deal with premicellar aggregation, it has shown that the dissociation time of micelles remains very large even below the cmc.

Our Kramers-like approach, as already mentioned in Sec. [I] relies on two assumptions, which are violated near the edges of the premicellar region. The first assumption, of a high free-energy barrier between the metastable and stable states, is valid in almost the entire region except close to the lower edge, $c_{2}$, where the barrier may become of order $k_{\mathrm{B}} T$ only. (See Table I) The resulting short lifetimes, though not accurately accounted for by the theory, are of little interest. The second assumption, of a large free-energy difference between the two states, holds in nearly the entire range as well, except very close to the upper edge, $c_{3}$, where, by definition, the free-energy difference vanishes. The free-energy difference, in units of $k_{\mathrm{B}} T$, becomes large quickly as $\phi$ gets smaller than $c_{3}$, since the considered mesoscopic subsystem of volume $V_{1}$ contains a large number of molecules (mostly monomers). In addition, correction of the theory near $c_{3}$ by considering a probability backflow from the monomeric to the aggregated state will only increase the stability of the latter. Therefore, the deficiencies of the theory at the edges of the premicellar region do not affect our main results.

It should be borne in mind, however, that the stability of premicellar aggregates imply also that high nucleation barriers may need to be overcome in order for them to form in the first place. Correspondingly, the more hydrophobic the surfactant, the higher these barriers are. (The issue of high nucleation barriers for micelle formation above the cmc has been underlined also in Ref. [20].) Hence, since both the preceding work [10] and the current one have assumed full equilibration, their applicability within reasonable time scales might require in practice either reduction of nucleation barriers through heterogeneous nucleation or overcoming them by external means (e.g., agitation or sonication).

Finally, we have found narrow size distributions of premicellar aggregates, i.e., micelles below the cmc should be only slightly more polydisperse than their counterparts above the cmc. (See Fig. 2, This agrees with the monodispersity observed in experiment [6]. Thus, polydispersity does not pose a problem for the applicability of premicellar aggregation. 


\section{Acknowledgments}

RH would like to thank R. Metzler and the Technical University of Munich for their hospitality. This research was supported in part by the Israel Science Foundation (Grant No. 588/06).

[1] F. Evans and H. Wennerström, The Colloidal Domain, 2nd ed. (Wiley, New York, 1999).

[2] J. N. Israelachvili, Intermolecular and Surface Forces, 2nd ed. (Academic Press, London, 1992).

[3] J. Israelachvili, J. Mitchell, and B. W. Ninham, J. Chem. Soc. Faraday Trans. 72, 1525 (1976).

[4] B. Lindman and B. Brun, J. Colloid Interface Sci. 42, 388 (1973).

[5] R. Sabate, M. Gallardo, and J. Estelrich, Electrophoresis 21, 481 (2000).

[6] H. Zettl, Y. Portnoy, M. Gottlieb, and G. Krausch, J. Phys. Chem. B 109, 13397 (2005).

[7] X. Cui, S. Mao, M. Liu, H. Yuan, and Y. Du, Langmuir 24, 10771 (2008).

[8] R. Barnadas-Rodriguez and J. Estelrich, J. Phys. Chem. B 113, 1972 (2009).

[9] A. D. Mackie, A. Z. Panagiotopoulos, and I. Szleifer, Langmuir 13, 5022 (1997).

[10] R. Hadgiivanova and H. Diamant, J. Phys. Chem. B 111, 8854 (2007).

[11] R. Zana, in Dynamics of Surfactant Self-Assemblies: Micelles, Microemulsions, Vesicles and Lyotropic Phases, edited by R. Zana (Taylor \& Francis, Boca Raton, FL, 2005).

[12] E. A. G. Aniansson and S. N. Wall, J. Phys. Chem. 78, 1024 (1974).

[13] E. A. G. Aniansson and S. N. Wall, J. Phys. Chem. 79, 857 (1975).

[14] E. A. G. Aniansson, S. N. Wall, M. Almgren, A. K. Shchekin, H. Hoffmann, I. Kielmann, W. Ulbricht, R. Zana, J. Lang, and C. Tondre, J. Phys. Chem. 80, 905 (1976).

[15] M. Almgren, E. A. G. Aniansson, and K. Holmaker, Chem. Phys. 191 (1977).

[16] M. Kahlweit and M. Teubner, Adv. Colloid Interface Sci. 13, 1 (1980).

[17] F. K. Gottberg, K. A. Smith, and T. A. Hatton, J. Chem. Phys. 108, 2232 (1998).

[18] N. A. M. Besseling and M. A. Cohen Stuart, J. Chem. Phys. 110, 5432 (1999).

[19] F. M. Kuni, A. P. Grinin, and A. I. Rusanov, Colloid Journal 63, 197 (2001).

[20] I. A. Nyrkova and A. N. Semenov, Macromol. Theory Simul. 14, 569 (2005).

[21] H. A. Kramers, Physica 7, 284 (1940).

[22] P. Hänggi, P. Talkner, and M. Borkovec, Rev. Mod. Phys. 62, 251 (1990).

[23] H. J. Woo, C. Carraro, and D. Chandler, J. Phys. Chem. B 108, 6778 (2004).

[24] J. S. Langer, Ann. Phys. N. Y. 54, 258 (1969).

[25] For $F^{\prime \prime}\left(m^{\max }\right) \leq-2$ this result evidently breaks down. In the representative examples of Sec. III we have $\left|F^{\prime \prime}\left(m^{\text {max }}\right)\right|<0.1$. One can find examples where $\left|F^{\prime \prime}\right|$ approaches 2 , yet this occurs only close to $c_{3}$, the upper edge of the metastable region, where the Kramers-like approach becomes inappropriate. Technically, the divergence can anyway be avoided by reducing the value of the parameter $\Delta$ in Eq. (12). 